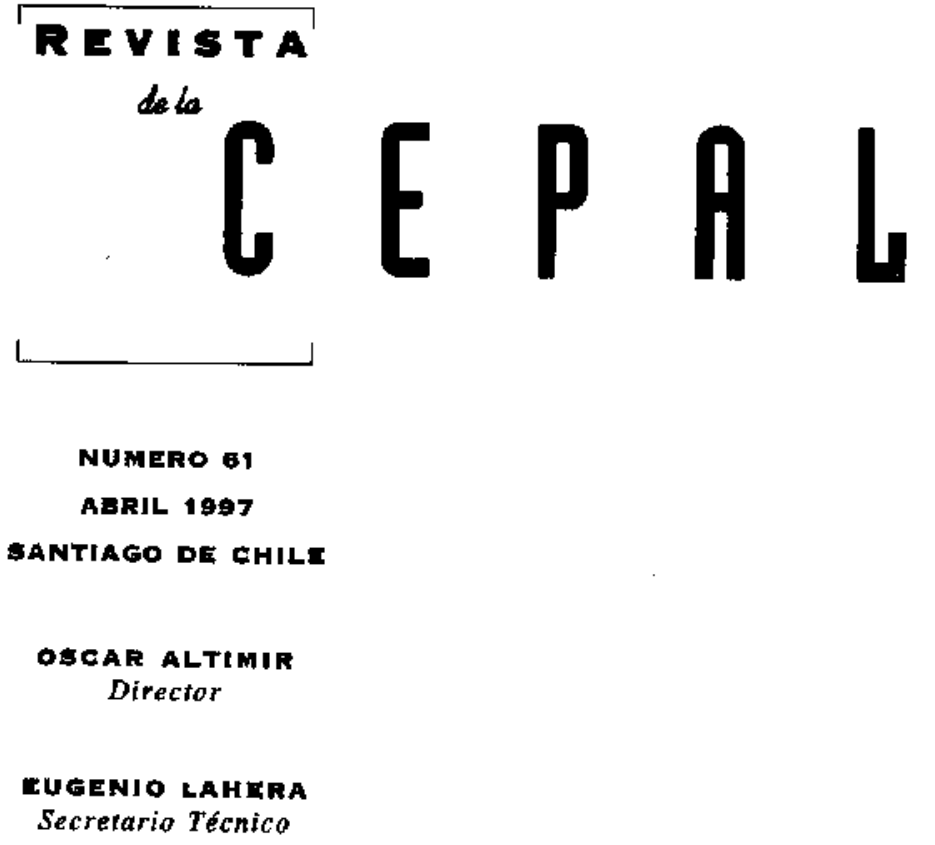

Secretario Técnico

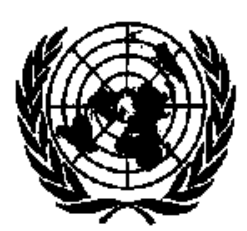

NACIONES UNIOAS 
Tres formas de coordinación social

Norbert Lechner

Brechas sociales en Colombia

Juan Luis Londoño de la Cuesta

Los Estados Unidos al rescate: la asistencia flnanciera a México en 1982 y 1995

Nora Lustig

El réglmen de convertibllidad y el sistema bancario en la Argentina Alfredo F. Calcagno

Exportaciones de manufacturas: desafíos para las pequeñas economías de América Latina

Rudolf M. Buitelaar, Pitou van Dijck

¿Por qué las inversiones en el transporte público no reducen la congestión de tránsito urbano?

lan Thomson

Nolas sobre la mediclón de la pobreza según el método del ingreso

Juan Carlos Feres

La politica fiscal y el ciclo económico en Chile

Carlos Budnevich, Guillermo Le Fort

Evaluación de la política de bienes de capital en la Argentina

Pablo Sirlin

Reestructuraclón de los grupos industriales brasileños entre 1980 y 1993

Ricardo $M$. Ruiz

Reestructuración productiva y cambio territorial: un segundo eje de Industrlallzación en el norte de México

Tito Alegría, Jorge Carrillo, Jorge Alonso Estrada

Publicaciones recientes de la CEPAL 


\section{¿Por qué las inversiones en el transporte público no reducen la congestión de tránsito urbano?}

\section{lan Thomson}

Unidad de Trunsporte, División de Comercio Internacional, Transporte y Financiamiento CEPAL
Existe congestión del tránsito urbano en la mayoría de las regiones del mundo, incluida América Latina. Entre las medidas destinadas a remediarla, en muchas ciudades se han construido líneas de trenes suburbanos o metros. Sin embargo, éstas han ayudado poco o nada, como lo confirman investigaciones según las cuales las inversiones en el sistema de transporte público por sf solas son ineficaces para resolver ese problema. En este artículo se plantea que al inaugurarse una nueva línea de metro, o sistema semejante, se transfieren a ella muchos viajeros que antes se desplazaban en buses, y unos pocos que antes utilizaban el automóvil. Esto libera espacio vial en las horas de máximo movimiento, el que es aprovechado por otros viajeros, quienes se cambian de horario o de ruta para ocuparlo. Además, los estacionamientos desocupados por las pocas personas que se cambian del automóvil a los flamantes trenes, son ccupados por vehículos con personas que antes utilizaban transporte público, por no tener dónde dejar sus automóviles. Al cabo de poco tiempo, en los períodos de más moviniento, aunque los nuevos trenes viajen llenos, la congestión de tránsito permanece virtualmente igual que antes. Esta secuencia muestra que los metros y sistemas semejantes generan sólo una fracción de los beneficios que podrían producir. Para que rindan más a la comunidad, deberían coordinarse con otras medidas, una de las cuales sería la de restringir más el estacionamiento en las zonas cercanas a las estaciones en el centro de las ciudades y en otras zonas comerciales. 
I

\section{Las expectativas y la realidad}

Lo que vemos en las calles nos hace dudar de que las mejoras en los sistemas de transporte público reduzcan la congestión de tránsito. Las ciudades con los sistemas más extensos de transporte público, como Londres o París, no se destacan por tener calles poco congestionadas. $Y$ en América Latina no parece que los metros y sistemas semejantes hayan aminorado la congestión.

Por otro lado, entre las razones más aducidas para justificar las inversiones en metros o sistemas semejantes está precisamente la de que ayudarían a disminuir la congestion. Hay expectativas de menor congestión en los anteproyectos pertinentes, en los pronunciamientos de técnicos y políticos y hasta en las evaluaciones de proyectos efectuadas por equipos de consultores. En Santiago de Chile, por ejemplo, se previó que en 1980 el metro iba a transportar 202330 pasajeros en la hora de más demanda y que de ellos unos 95800 se transferirían de vehículos particulares (más que del transporte colectivo). ${ }^{1}$ Es decir, se esperaba que un $47.4 \%$ de los usuarios del metro se cambiarían a él desde el transporte privado.

Quizás los consultores, principalmente extranjeros, contratados para evaluar el entonces propuesto metro de Santiago, fuesen más optimistas que sus con-trapartes en otras ciudades respecto a la posibilidad de interesar a los automovilistas en el nuevo sistema, pero había optimismo también en otras partes. Algunos investigadores que examinaron los objetivos de los diferentes proyectos para el metro señalaron que los objetivos principales del proyecto, en relación con la situación del transporte y de la ciudad en el momento y a futuro, incluían mejorar la fluidez y velocidad de los desplazamientos, remediar la congestión de tránsito, atender a demandas sociales, revitalizar centros y corregir la aglomeración (Figueroa, 1986), y que se creía ampliamente que un metro paliaría la congestión al reducir el número de buses en la calle y posiblemente atraer a algunos automovilistas (Allport y Thomson, 1990).

Los no especialistas en transporte urbano considerarán lógico que con la inauguración de un metto se redujera la congestión de tránsito en las calles del mismo corredor. Muchos especialistas creerían lo mismo. Sin embargo, como se intenta demostrar aquí, lo que se da en realidad es un fenomeno aparentemente ilógico.

\section{II}

\section{Nuestra tesis básica y la metodología para la evaluación socioeconómica de los metros}

La tesis básica del presente trabajo se puede resumir en muy pocas palabras:

i) Una mejora en el sistema de transporte público aumenta muy poco el número total de usuarios de ese sistema, siendo su impacto principal una redistribución de la demanda hacia los componentes mejorados desde aquéllos no mejorados que compiten con los primeros.

\footnotetext{
${ }^{1}$ Estimado sobre la base de los cuadros en las páginas 98 y 100 de SOFRETU/BCEOM/CADE, 1968.
}

ii) Los relativamente pocos automovilistas que se cambian al componente mejorado del sistema de transporte público liberan espacio vial y estacionamientos, los que son aprovechados por otras personas, entre ellas algunas que antetiormente utilizaban el transporte público, de modo que el nivel de congestion vuelve a los niveles que había exhibido antes de que se mejorase la red de transporte público.

iii) La reducción del número de pasajeros de autobuses también podrá liberar espacio vial (aunque no estacionamientos), lo que incentivará una redistribución temporal de los viajes en automovil; de esa manera el 
espacio liberado en el período de demanda más intensa vuelve a ocuparse, dejando más libres las calles en los momentos cercanos a ese período.

Diversos estudios ${ }^{2}$ dejan bastante en claro que los metros o sistemas semejantes no reducen la congestión de tránsito. Esta conclusión invalida las de otras investigaciones que suponen que sí la aminoran y que, por lo tanto, traen beneficios ambientales y otros. Por ejemplo, parecería equivocado afirmar que "en todos los países del mundo hay un clare reconocimiento de los efectos negativos que la congestión vehicular provoca sobre el medio ambiente $y$, en consecuencia, se ha tomado conciencia de los beneficios de los trenes livianos, cuyas ventajas son, la limpieza, la seguridad,..." (Jorge, 1994). Si los trenes livianos o los de más peso no reducen la congestion vehicular, sus beneficios ambientales serían nulos.

La tesis propuesta tiene algunas implicaciones para la metodología de la evaluación socioeconómica de los metros. El que un metro o sistema semejante no reduzca la congestión no significa, desde un punto de vista socioeconómico, que no sea conveniôte su implantación; pero sí significa que los beneficios que traerían los proyectos de implantar el nuevo sistema corresponderían a las diferencias de costo entre el transporte de los usuarios por el metro y por los medios colectivos usados anteriormente. A veces será necesario tomar en cuenta también la posibilidad de que el metro permita elevar el número total de viajes efectuados.

En cásos típicos, será bastante fácil hacer los cálculos correspondientes; es decir, si se supone que los metros no tienen influencia importante sobre la congestión de tránsito, su evaluación socioeconómica será mucho más sencilla que si se trata como variable el grado de congestión.

\section{III}

\section{Profundización de algunos aspectos}

\section{de la tesis propuesta}

\section{Las consecuencias de los cambios en el uso del suelo causados por el metro}

Los metros mejoran la accesibilidad de algunas partes del area urbana, especialmente en los alrededores de las estaciones. Esto eleva el valor de las propiedades $y$ puede poner en marcha tendencias a la densificacion en las zonas de las estaciones, por ejemplo, mediante la construcción de edificios altos de departamentos habitacionales y especialmente de oficinas.

Existen modelos matemáticos que incluyen tanto la simulación de los patrones de transporte como el uso del suelo. Se han aplicado en algunas ciudades latinoamericanas, como São Paulo. Sin embargo, aunque son intelectualmente interesantes y a veces elegantes desde el punto de vista matemático, su utilidad es muy limitada, porque son casi imposibles de calibrar. Por otra parte, y aunque en la práctica no sea posible cuantificarla con anterioridad, es innegable en principio la existencia de una relación bidireccional entre el sistema de transparte y el uso del suelo.

\footnotetext{
${ }^{2}$ Foster y Beesley, 1963; Younes, 1995; Allport y Thomson, 1990; Bamford y Allport, 1990.
}

Esa relación no siempre se manifiesta, por diversas razones, entre las cuales se incluyen los planos reguladores preestablecidos que enmarcan las acciones naturales del mercado de propiedades. Por lo tanto, la densificación del uso del suelo no siempre ocurre, al menos a escala significativa (por ejemplo, en Río de Janeiro y San Francisco), pero sí se ha dado en lugares como los suburbios de Londres en los primeros decenios del siglo veinte y más recientemente en Santiago de Chile, Hong Kong y Toronto, a veces por la acción natural del mercado y otras veces por un proceso de planificación del sector privado o del públi$\mathrm{cos}^{3}$

Cuando el metro incentiva el desarrollo comercial atrededor de las estaciones, es normal que una mayoría de las personas que trabajan allí, y de sus clientes y otras personas a quienes atienden directamente, usen el metro para ir y venir de tales sitios. Sin embargo, un número significativo de esas personas se desplazan en automóvil, agravando así la congestión. En Santia-

\footnotetext{
${ }^{\exists}$ Véanse, por ejemplo, Allport y Thomson (1990), y tanbién Knight, 1980.
} 
go, la concentración de los viajes motorizados que no son sobre rieles es superior en la zona de influencia del metro que en la ciudad en general y, con más razón aún, que en los sectores donde no hay metro (cuadro 1).

Así; aunque podría parecer ilógico, el metro traería como consecuencia la generación de un mayor número de viajes en automóvil a su propia zona de influencia. Sin la construcción del metro, es probable que esos viajes en automovil se efectuaran en otras partes de la ciudad. Pero es igualmente probable que en esas otras partes la densidad de desarrollo comercial hubiera sido inferior a la del corredor del metro y que allí esos viajes hubieran creado menos congestión.

Esta consecuencia indirecta del metro -la de estimular el uso del automóvil en las zonas que atiende-podría encararse con la imposición de controles estrictos sobre el estacionamiento en la calle y fuera de ella, incluso en los propios edifícios nuevos que se construyen; sin embargo, en América Latina esto se ha hecho poco. Es frecuente, por ejemplo, que se fije un número mínimo de estacionamientos por metro cuadrado o por persona empleada, siendo que lo que debería hacerse es fijar un número máximo (véase $\mathrm{El} \mathrm{Mercu-}$ rio, 1996).

\section{La demanda latente de viajes en automóvil}

Si una persona dispone de un automóvil es muy probable que lo use para sus viajes, aunque esta opción no le signifique ahorro en tiempo o costos monetarios comparada con la de viajar por transporte público. Puede preferir el automóvil por una variedad de razones, como la flexibilidad para escoger su propio horario de regreso, las posibilidades de llevar paquetes, la mayor protección contra el clima, la mayor privacidad o la oportunidad de escuchar la estación de radio que prefiera.

Los modelos de simulación del transporte reflejan de distintas maneras esa preferencia por desplazarse en automóvil por razones subjetivas. Una opcion es ta de determinar factores de ajuste que se cuantifican mediante el proceso de calibración del módulo de particion modal. En términos sencillos, lo que se hace es escoger valores esencialmente monetarios que al ser usados para modificar los costos de transporte por el automovil permiten reproducir lo mejor posible la partición modal observada; los factores representarían las preferencias reveladas de los viajeros. Otro método, usado con más frecuencia en los úitimos años, es el de utilizar encuestas para determinar las preferen-
CUADRO I

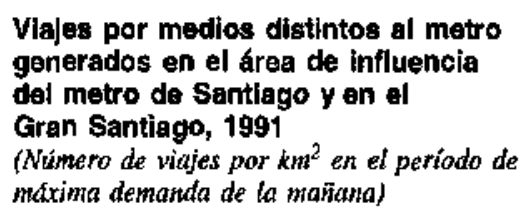

\begin{tabular}{lrr}
\hline Medio de transporte & $\begin{array}{r}\text { Avea de influencia del metro } \\
\text { de Santiago }\end{array}$ & $\begin{array}{c}\text { Gran } \\
\text { Santiago }\end{array}$ \\
\hline Viajes de automóyiles & 816 & 492 \\
Viajes de buses (en automóviles & & \\
equivalentes) & 206 & 122 \\
Viajes de taxis & 21 & 12 \\
Viajes de taxis colectivos & 33 & 14 \\
$\Sigma$ & 1076 & 640 \\
\hline
\end{tabular}

Fuente: Estimación propia a base de datos obtenidos en Encuesta origen destino de viojes del Gran Santiago 1991, Comisión de Planificación de Inversiones en Infraestructura de Transporte (sin fecha); Pontificia Universidad Catolica de Chile, Instituto de Economía (1993); Empresa de Transporte de Pasajeros Metro S.A. (varios afios), y consultas telefonicas a la misma empresa.

a El área de influencia del Metro se define como una faja de cinco cundras (consideradas equivalentes a 500 metros) a lo largo de las líneas.

cias declaradas de los viajeros entre los diferentes medios para viajar, definidas en términos de costo, tiempo y otros atributos.

Es frecuente encontrarse con que la demanda de viajes en automóvil a las zonas comerciales más desárrolladas de las ciudades supera la capacidad de los estacionamientos; esto se incorpora en los modelos de diferentes maneras. Si la inauguración de un metro, u otra mejora en el sistema de transporte público, tuviera como consecuencia inicial un desplazamiento significativo de la demanda desde el automovil hacia el sistema mejorado, se liberarían lugares para estacionar. Sin embargo, debido a la demanda no satisfecha por viajar en automóvil (por no haber habido un número suficiente de estacionamientos), los lugares liberados no permanecetian vacíos por mucho tiempo porque otras personas, que antes viajaban por transporte público, utilizarían ahora el automóvil para aprovecharlos. Así, el número de viajes en auto sería el mismo de antes, aunque probablemente tendrían diferentes zonas de origen.

En otras palabras, la inauguración de un metro sobre un eje de una ciudad podría desembocar en un mayor número de viajes en automóviles sobre otros ejes. Este resultado se puede simular mediante un modelo normal de planificación del transporte; sin embargo, hay otros efectos comparables que es imposible simular a través de la gran mayoría de esos modelos. 


\section{Las preferencias de horarios y el espacio viai}

El metro debería ocasionar al inicio una baja de congestión en las vías paralelas a él, debido tanto a una pequeña disminución del tránsito de automóviles como a una de mayores proporciones en el de buses. Sin embargo, esa baja también podrfa ser transitoria.

Muchas personas, entre las que me incluyo, no viajan en las horas de máximo movimiento, por la demora y la tensión o frustración que causa el tránsito agudamente congestionado frecuente en tales momentos. Naturalmente, les acomodaría viajar a esa hora, pero salen un poco antes o un poco después para poder desplazarse en momentos en que el tránsito sea algo más fluido.

Sin embargo, con la inauguración de un nuevo metro o sistema semejante la congestión de tránsito en las calles del corredor del metro disminuiría, con lo cual se alentaría a aquellas personas que, como yo, viajaban un poco antes o un poco despues de los períodos de demanda máxima, a cambiarse a los horarios de más movimiento.

Como resultado, la congestion sería casi tan aguda como antes en el momento de más tránsito y se reduciría más o menos perdurablemente en los períodos inmediatamente anteriores y posteriores. Con esto se volvería más aguda la concentración de la distribución de frecuencias en el tiempo.

\section{La convenlencia politica}

Es útil agregar aquí algunas palabras sobre la conveniencia, desde el punto de vista de los intereses de los políticos locales, de realizar proyectos de metros, para ayudar a entender por qué se construyen nuevos sistemas de transporte público que poco o nada hacen para reducir la congestión.

Saivo contadas excepciones, los alcaldes, intendentes y otros que promueven la construcción de los metros mejoran sus perspectivas políticas al hacerlo. Normalmente, la ciudad en que se construye un metro paga sólo una pequefia parte del costo de inversión en él, pero casi la totalidad de los beneficios que éste genera permanecen dentro de esa zona metropolitana (véase Thomson, 1985). Los ciudadanos, evidentemente, ganan más que pierden y tienden a agradecer a sus representantes políticos que hayan intervenido en la decision de construir el metro. Los que pierden son los demás habitantes del país, que contribuyen a sufragar los costos de inversión aunque no perciben ni siquiera una parte mínima de los beneficios.

Por lo tanto, los políticos locales tienden naturalmente a promover la construcción de un metro en su ciudad, justificándola con argumentos que parecen logicos a primera vista, pero que no resisten un análisis más a fondo.<smiles>[AlH2]</smiles>

\section{El efecto del metro sobre la congestion:}

\section{algunos casos concretos}

A continuación se resumirán algunas conclusiones importantes para nuestro análisis que surgen de diversos estudios realizados para determinar el efecto de un metro, u otro sistema de transporte masivo o rápido, sobre la congestión.

Distinguiremos ante todo entre dos tipos de repercusiones de los metros sobre los volúmenes de tránsito callejero: el impacto directo, representado por el número de viajeros que se cambian al metro, y el impacto indirecto, representado por cambios en el comportamiento de los viajeros ocasionados por el impacto directo, mediante los cuales es posible estimular transferencias hacia el transporte privado. Hacemos esta distinción porque normalmente las empresas de metros están en condiciones de estimar los impactos directos a través de encuestas a los usuarios, pero muy pocas veces ellas, $u$ otras entidades, saben mucho sobre los impactos indirectos.

\section{Los resultados de los análisls por modelos de simulactón}

El Laboratorio de Investigación en Transporte del Reino Unido ha investigado mediante un modelo de simulación los efectos en el volumen de tráfico de automóviles que causan distintos niveles de inversión en transporte público sobre rieles. Se modeló una ciudad de tamaño mediano, se postuló la construcción de un me- 


\begin{tabular}{lcc}
\hline & $\begin{array}{c}\text { Cambio porcentual de automóvil/kilómetros en } \\
\text { comparación con el caso base }\end{array}$ \\
\hline Metro en tres corredores radiales & $\begin{array}{c}\text { Período de máxima denanda } \\
\text { Periodo de menor demanda }\end{array}$ \\
Metro en seis corredores radiales & -1.3 & -0.3 \\
Metro en seis corredores radiales, tarifas x $50 \%$ & -2.6 & -0.6 \\
\hline
\end{tabular}

Fuente: The Chartered Institute of Transport, 1996, anexo B.

tro o sistema de trenes livianos - Rail Transport (LRT) - en corredores radiales, y se evaluó además el impacto adicional de modificaciones tarifarias (véase los resultados en el cuadro 2).

No tenemos información sobre la longitud o el costo de los sistemas de metro y LRT modelados; sin embargo, es bastante evidente que inversiones muy importantes en la construcción de tales sistemas causan reducciones mínimas en el uso del automóvil privado. Aun así, los pequeños cambios calculados en el uso de este vehículo podrían llevar a sobreestimar los cambios acompañantes en la congestión, puesto que es altamente improbable que el modelo usado (del cual no tenemos antecedentes) sea capaz de simular la redistribución temporal de los viajes en el período de máxima demanda, o entre ese período y las demás horas del día, a raíz de cambios diferenciales en la liberación de espacio vial por la transferencia de algunos viajes al metro $0 \mathrm{LRT}$.

Otros estudios llevados a cabo en Gran Bretaña indican resultados algo parecidos. La implantación en Londres de un amplio programa de construcción de líneas férreas, principalmente subterráneas -incluido un nuevo ferrocarril sobre el eje este-oeste por el centro de la ciudad, una extensión de la línea Jubilee del subterráneo, una ampliación del LRT de Docklands y un sistema de tranvía en el suburbio de Croydon, entre otros-, reduciría el porcentaje en persona/kilómetros correspondiente a viajes en automóvil del $52.4 \%$ al $47.6 \%$, siempre que además las frecuencias de los servicios de buses mejorara en un $20 \%$, que se perfeccionara el sistema de control de tránsito y se garantizara un control rígido sobre el estacionamiento en las calies y avenidas de la ciudad. ${ }^{4}$ Otro estudio, también de Londres, estimó que la aplicación de una estrategia de mejoras en las frecuencias de los servicios de buses y

\footnotetext{
4 Véase The Chartered Institute of Transport, 1996, anexo B.
}

de trenes, junto con una ampliación del sistema de preferencia para los buses en el tránsito, reduciría los vehículos $/ \mathrm{km}$ en el centro en $1 \%$, y en $2 \%$ en el resto de la ciudad. Si al mismo tiempo se invirtiesen cuantiosas sumas en el sistema ferroviario, las cifras cambiarían a 4 y $5 \%$, respectivamente.

Segun el informe de The Chartered Institute of Transpout (1996, anexo B) todos los estudios pertinentes señalan que las acciones destinadas a mejorar la situación competitiva del transporte público probablemente sólo tengan efectos menores sobre el uso del automóvil. Por otra parte, sus autores consideran que los modelos podrían subestimar el efecto que tendrían las mejoras en el transporte público sobre el tráfico automovilístico, por no tomar adecuadamente en cuenta los aspectos cualitativos más bien que los cuantitativos (Ibid., p. 49).

\section{La línea Victoria del subterráneo de Londres}

La línea Victoria, que corre entre los suburbios del centro-este londinense y la zona céntrica, posteriormente extendida hacia el sur, fue planificada en la década de 1960 e inaugurada a fines de ella. El análisis de costo-beneficio llevado a cabo para comprobar la factibilidad económica de su construcción fue considerado un ejemplo en esa época y todavía se hace referencia a él como un estudio clásico (Foster y Beesley, 1963, pp. 46 a 78). Entre los objetivos declarados de la construcción de la línea se hallaba la reducción de la congestión callejera, y se esperaba que la posibilidad de desplazarse más rápidamente a través de ella disminuyera el volumen de automóviles en las calles y favoreciera la fluidez del tránsito de los buses. Un $35 \%$ de los beneficios de la línea, según el análisis mencionado, se debería a la reducción de dicho volumen, al disminuir en unos $\mathbf{8} 000$ los viajes direccionales de automóviles por día.

En realidad, sólo 5500 de los usuarios de la nueva línea se cambiaron a ella desde el automovil, y 1400 
de éstos siguieron usando el automovil como medio de acceso al subterráneo. El investigador Bassem Younes sugiere que la desocupación de espacio en las calles, ocurriđa inmediatamente después de la inauguración de la línea, puede haberse invertido a través del aprovechamiento de ese espacio por una demanda latente de viajes en automovil (Younes, 1995, pp. 333 a 336), y concluye que el impacto de la línea Victoria sobre el tránsito de automóviles fue muy marginal y que las calles continuaron llevando casi el mismo volumen de tráfico que antes.

\section{Berlín occldental}

Younes analizó también la extensión del metro de la entonces ciudad de Berlín occidental hasta el distrito de Spandau entre 1980 y 1984 ; en este caso, como en el Iondinense, uno de los objetivos era rebajar la congestión de tránsito en las calles. Concluyó que en Berlín se comprobaba una vez más que las mejoras al transporte público no conducían necesariamente a una reducción significativa e importante del uso del vehí" culo motorizado (Younes, 1995, pp. 333 a 356). ${ }^{5}$

Llegó a esa conclusión al estudiar los resultados de una encuesta efectuada por el Departamento de transporte y obras públicas de Berlín. Sin embargo, en ese caso particular es posible interpretar los resultados de una manera diferente a la suya. En Spandau, entre 1979 y 1985 , la proporción de viajes efectuados por automóvil aument 6 modestamente, de 42.6 a $43.2 \%$, mientras que la de viajes por transporte público subió un poco más, de 25.3 a $27.3 \%$. En el distrito comparable de Lichtrenade, donde no hubo cambios en la red del Metro, la proporción correspondiente a los automóviles subió de 43.7 a $52.4 \%$ y la correspondiente al transporte público bajo de 31.8 a $24.6 \%$. Vemos así que con las mismas cifras básicas que usó Younes se puede llegar a una conclusión diferente: que la extensión del Metro a Spandau sí habría tenido un impacto sig. nificativo sobre el uso del autemovil privado. Cabría concluir entonces que la ampliación de la red del metro de Berlín occidental a Spandau sí habría contribuido a reducir la congestión de tránsito.

\section{Stuttgart}

Este otro caso estudiado por Younes se refiere a una prolongación de la línea $1^{\text {a }}$ de la red del ferrocarril sub-

\footnotetext{
5 Younes usa el término motor vehicle, aunque es evidente que se refiere especificamente al automóvil, más que a otras formas de vehículos motorizados que en principio incluyen hasta los coches
} motores de los matros. urbano, entre Schwabstrasse y Vaihingen/Böblingen, la que fue inaugurada en septiembre de 1985. También aquí uno de los objeivos del proyecto era frenar el uso creciente del automóvil. La tarea de Younes se facilitó porque la autoridad metropolitana había realizado un estudio de tráfico en el eje de influencia de la extension, tanto antes como después de su puesta en servicios.

A pesar de la extensión de la Línea, el tránsito sobre las calles de la ciudad creción más dentro de la zona de influencia de la extensión que en el resto de la ciudad (cuadro 3). El crecimiento fue en general inferior más hacia el centro, por la saturación de las vías y las restricciones impuestas por la disponibilidad de estacionamientos.

Younes concluyó que el impacto de la extensión del ferrocarril suburbano se hizo sentir dentro del sistema de transporte público especialmente por transferencias desde los buses a los trenes, y que cualquier capacidad vial liberada fue aprovechada por una demanda latente.

Es evidente también que la extension ferroviaria permitió más viajes hacia el centro de la ciudad y que implícitamente habría contribuido a hacer más viable la parte céntrica de la ciudad, especialmente como zona de concentración de empleos.

\section{Ciudades de paises en desarrolto}

Un estudio llevado a cabo por el Laboratorio de inves. tigación en transporte del Reino Unido expresa que se ha encontrado poca evidencia de que haya una relación entre la construcción de un metro y la reducción de los volúmenes de tránsito, y que la mayoría de los usuarios de los sistemas de transporte sobre rieles en los países en desarrollo provienen de otras formas de transporte público.

Otro estudio británico que analiź el efecto sobre la congestión de los sistemas de transporte masivo en los países en desarrollo (Allport y Thomson, 1990), en general concluyó que en casí todos los corredores de transporte había desde antes de la construcción del metro una congestión entre moderada y aguda. En la gran mayoría de casos, la mejoría parece haber sido poca o inexistente, y en muchos casos la congestión se agrav6. En algunas instancias habra habido una mejora evidente inmediatamente después de la inauguración del metro. pero el beneficio había sido transitorio. Este último acontecimiento es consistente con nuestra tesis.

De 12 casos presentados (recuadro I), solamente en tres se atribuyó al metro o al LRT (es decir, a sistemas de transporte público sobre rieles que fuesen li- 
vianos y rápidos) una reducción significativa en la congestión de tránsito. Otro estudio concluyó que, en general, la proporción de los ocupantes de automóvi- les que se transferirian a un metro inaugurado en el mismo corredor fluctuaría entre 0 y $4 \%$ (Bamford y Allport, 1990).

CUADRO 3

Cambios en los volúmenes de transporte privado sobre las vías camineras, antes y después de prolongarse la red de tren suburbano de Stuttgart (Línea 13)

\begin{tabular}{llcc}
\hline & Anillo & $\begin{array}{c}\text { Aumento del } \\
\text { tráfico entre mayo de } \\
1984 \text { y abril de } 1986 \\
(\%)\end{array}$ & $\begin{array}{c}\text { Aumento de tráfico } \\
\text { entre octubre de 1984 y } \\
\text { octubre de } 1986 \\
(\%)\end{array}$ \\
\hline $\begin{array}{l}\text { En el área de influencia } \\
\text { de la prolongación }\end{array}$ & $\begin{array}{l}\text { Interior } \\
\text { Exterior }\end{array}$ & 3.6 & $\ldots$ \\
En toda la ciudad & Exterior & 13.3 & 11.5 \\
\hline
\end{tabular}

Fuente: Younes, 1995, p. 350. (Se han corregido algunos errores menores en el cuadro original).

\section{Recuadro 1}

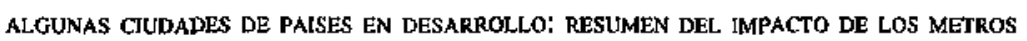
SOBRE LA CONGESTION DE TRANSITO

El Caito Ningún impacto observable.

Calcuta Ningún impacto.

Hong Kong La propiedad de los automóviles bajó en la época de la inauguración del metro, pero la causa fute un aumento de los impuestos, más que el metro. Un 16\% de los pasajeros autobuseros se transfirieron al metro, pero luego los flujos de buses subieron y la congestión permaneció igual que antes.

Manila Hubo una reducción en la congestion, debida probablemente al mevo LRT y a una recesión económica.

Ciudad de México La congestión causada por los automóviles siguió siendo aguda, aunque la propiedad de los automoviles bajó debido a una recesión económica. La velocidad de los buses es relativamente alta supuestamente por la instalación de pistas exclusivas y no tanto gracias al metro.

Porto Alegre La congestión no era severa antes de la inauguración del tren suburbano ni lo fue después.

Pusán El metro probablemente contribuyó a paliar la congestión de tránsito.

Río de Janeito Los flujos de los buses se redujeron solamente un poco, por lo tanto no pudo haber habido mucho impacto sobre la congestión.

Santiago La congestion siguio siendo grave sobre el eje principal este-oeste, y los flujos de buses se mantienen cerca de los niveles máximos posibles.

São Paulo Los flujos de buses bajaron en 500 por hora y sentido en cada corredor; inicialmente nubo una menor congestión, pero luego ésta volvió a ser grave, alcanzando a nuchas zonas.

Seúl La congestión era seria, generalizada y en rápido aumento. El sistema de transporte masivo no tuvo un impacto observable sobre los volúmenes de tránsito o los flujos de buses.

Túnez No se esperó ningún impacto sobre la congestión, lo que se comprobó en la práctica.

Fuente: Allport y Thomson, 1990, cuadro 8.1. El comentario sobre las pistas exclusivas, en el caso de Ciudad de México, es propio. 


\section{Una forma de transporte público que sí}

\section{atrae a los automovilistas}

\section{El rechazo de los automovilistas a los buses regulares}

La experiencia mundial indica que mejorar los sistemas de buses comunes y corrientes no atrae a los automovilistas. Los sistemas corrientes sobre rieles (metro, tranvia y otros) les interesan más, pero no demasiado. Unicamente las variantes del transporte público que ofrecen al automovilista un grado superior de comodidad han logrado sacar a una cantidad significativa de automovilistas de sus vehículos privados y agregarlos a las filas de los usuarios del transporte público.

En paises más adinerados, tales medios públicos más cómodos podrían correr sobre rieles. Por ejemplo, los metros de ciudades estadounidenses como San Francisco o Washington ofrecen condiciones de viaje bastante agradables y sus estaciones en los suburbios disponen de amplias playas de estacionamiento, planificadas para viajes bimodales que combinan medios de transporte privado y colectivo. Esta es una opción interesante para el automovilista. Sin embargo, en ningún país latinoamericano es social o políticamente aceptable financiar, necesariamente con fondos públi$\cos$, la construcción de un metro para satisfacer los gustos de la elite.

\section{Buses de lujo}

Existe la opción de autorizar la operación de servicios de buses de una categoría superior, que ya circulan por las calles y avenidas de ciudades como Buenos Aires, Córdoba, Ciudad de Guatemala, Río de Janeiro, São Paulo, Santafé de Bogotá y otras. Esos buses suelen disponer de asientos cómodos, reclinables, de sistemas de aire acondicionado y de música ambiental; además, no llevan pasajeros de pie. En Buenos Aires y algunas ciudades brasileñas viajar en ellos cuesta al usuario unas cuatro o cinco veces más que en un bus común. A pesar del precio los servicios de categoría superior atraen cantidades suficientes de usuarios como para convertirlos en una actividad rentable. Cabe sefialar, sin embargo, que la situación financiera de las empresas operadoras es muy susceptible a cambios en el ambiente económico. Los problemas económicos de
Brasil en los años ochenta afectaron mucho la popula ridad de los frescóes de Río de Janeiro y es bastante probabie que el estancamiento de la economía argentina a mediados de los noventa esté repercutiendo sobre los balances de las empresas que operan los servicios "diferenciales" de Buenos Aires.

La creación y operación de los servicios de categoría superior normalmente cuestan poco o nada al sector público, y se han demostrado exitosos en atraer al automovilista privado. Por ejemplo, en Buenos Aires un $14 \%$ de los usuarios de los buses diferenciales habrían usado el auto particular como medio optativo (Vicente y Brennan, 1989). En Bogotá, 48\% de los usuarios de los buses ejecutivos provenían de familias con uno o más autornóviles, y $19 \%$ contaban con un autom6vil para su uso preferencial (Acevedo, 1989). En Amsterdam, 39\% de los usuarios de un servicio de buses expresos de lujo optaron por él en lugar de viajar en automóvil privado (OCDE, 1994).

Es evidente que los buses de categorfa superior pueden tener éxito en captar a personas que sin ellos habrian viajado por automóvil. Este sería un impacto directo (sección IV, subsección 1 de este artículo). Sobre el impacto indirecto no hay información; sin embargo, si no se toman medidas para controlarlo es bastante probable que los estacionamientos y el espacio vial liberados por el menor uso directo del automóvil sean aprovechados por otras personas que hagan el cambio opuesto, es decir, desde el transporte público al transporte particular. ${ }^{6}$

\section{El slstema de Curitlba}

En la ciudad paranense de Curitiba en Brasil no existe una categoria superior de transporte colectivo, si se toma como base de comparación la calidad media de dicho transporte en la ciudad; pero el sistema de transporte colectivo en general es de una calidad superior a la de casi cualquier otro en América Latina. En

\footnotetext{
6 Vease mayores antecedentes sobre la influencia de los buses de categorfa superior en la congestión, en Thomson (1996).
} 
Curitiba, los buses expresos corren sobre una red de vías exclusivas de unos $60 \mathrm{~km}$, las distintas líneas se hallan integradas entre sí y los habitantes -de todas las clases sociales- tienen una buena percepción del sistema. A pesar de las vías exclusivas, un viaje hecho en automovil particular tiende a demorar menos que el mismo viaje realizado por transporte colectivo (véase Buleze y otros, 1985, cuadro 12); de todos modos, un porcentaje importante de los usuarios del transporte colectivo rechazan la opción de viajar en automóvil: lo dejan en la entrada de sus casas y prefieren el bus.
Sin duda, algunos de dichos usuarios eligen el bus por razones de conveniencia, confiabilidad o costo, y otros por no contar con un lugar donde estacionar su vehículo. Sin embargo, no cabe negar que las cualidades intrínsecas de un sistema de transporte colectivo integrado y bien planificado durante muchos años puedan hacerlo suficientemente atractivo como para ser preferido por personas que, de no existir ese sistema, habrían viajado en automóvil. Lo que no se sabe es si la reducción directa de congestión que pudiera producirse se mantendría, o si sería compensada por la generación indirecta de otros viajes en automovil.

\section{VI}

\section{La importancia de las medidas para controlar el uso del automóvil particular}

\section{Principios básicos}

Si se observa la situación de la red vial en un corredor antes y después de la inauguración de un metro, vemos que éste, como impacto directo, atrae una cantidad de viajes que antes se efectuaban por bus y un número reducido de los que antes se hacian en automóvil, con lo cual aumenta el espacio vial disponible para otros usuarios de la red vial (gráfico 1). La relación que describe la oferta del espacio vial de que disponen estos últimos es representada por la curva $A c(0)$

GRAFICO I

Representación teórica de la ocupación de la red vial por no usuarios del metro

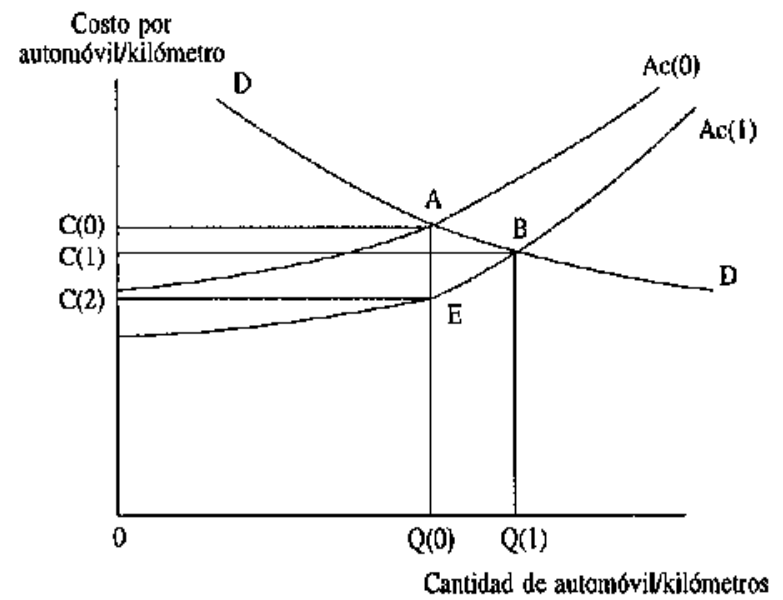

antes de la inauguración del metro, y por la $A c(l)$ después de su inauguración. La relación de la demanda de uso del espacio vial se define por la curva $D D$, cuya forma refleja la naturaleza elastica de esta demanda.

En el caso presentado en el gráfico no se contempla la aplicación de un régimen de tarificación vial, mediante el cual los usuarios de la vialidad pagarfan tasas o peajes que reflejasen la diferencia entre el costo medio y el costo social marginal de su ocupación de las yías. Tanto en la situación sin metro como en aquella con metro, se podrían generar beneficios socioeconómicos al aplicar tales tasas o peajes, reduciendo de esa manera los volúmenes de tránsito y la congestión.

En la primera situación, el volumen de tráfico es $O Q(0)$, a un costo medio de $O C(0)$. Una vez inaugurado el metro, ese volumen sube significativamente a $O Q(I)$ y el costo medio baja, en un monto relativamente inferior, a $O C(I)$. Los beneficios percibidos por los usuarios correspondientes se identifican por el área $C(O) A B C(I)$.

Sin embargo, si en la segunda situación fuera posible restringir el volumen de tráfico al existente antes de que se pusiera en marcha el metro, los beneficios percibidos ascenderían al monto considerablemente mayor de $C(O) A E C(2)$. Evidentemente, si fuera factible limitar el incremento de la ocupación de la red vial en términos de bienestar total sería conveniente hacerlo. 


\section{Medidas prácticas}

La necesidad de restringir los impactos indirectos es evidente, pero cómo hacerlo no lo es tanto. La cantidad optima de restricción varía en cada caso y en principio depende no solamente del número de viajes transferidos al metro o del espacio vial liberado, sino también de otros factores, como la gravedad de la congestión en el eje.

En términos prácticos, sin embargo, sería razonable tratar de reducir la capacidad de los estacionamientos en la zona de influencia de cada estación de metro en un número igual al número de motoristas y de automoviles que viajan a cada una de dichas zonas y que se hayan transferido al metro por el impacto directo de éste. Las reducciones correspondientes podrían cuantificarse al analizar los resultados de un modelo de simulación de transporte, lo que permitiría disminuir el número de estacionamientos junto con inaugurarse el metro.

Otra opción sería la de llevar a cabo una encuesta entre los usuarios del metro, una ver puesto en marcha éste, para determinar el número de pasajeros que anteriormente se desplazaban manejando automóviles. Esta última metodología tiene la ventaja de ser más precisa, pero desde el punto de vista político sería más complicada, porque significaría retirar estacionamientos de automovilistas que estarian acostumbrándose ya a disponer de ellos.
Son pocas las medidas prácticas que pueden adoptarse para minimizar la incidencia de los cambios en los horarios de los automovilistas que reprograman sus viajes para efectuarlos no ya en períodos cercanos al momento de mayor demanda, sino en el momento de mayor demanda mismo, para aprovechar la liberación de espacio vial generada por el impacto directo del metro. Una opción podría ser la de imponer controles sobre el horario de ingreso a los estacionamientos; pero eso sería difícil de llevar a cabo en la práctica, entre otras razones, por el hecho de que los viajes que cambiarlan de horario no tendrían como destino una sola zona, sino que estarían destinados más bien a una multiplicidad de áreas donde compartirían lugares de estacionamicnto con otros automovilistas, a cuyos viajes no se prevé aplicar medidas restrictivas.

En teoría, se podrían controlar los horarios de los viajes en automóvil mediante una versión sofisticada de la tarificación vial que vinculara el valor de la tarifa cobrada con la congestion existente a cada hora del día. Este tipo de instrumento en general permitiría adecuar los volúmenes de tránsito a la capacidad vial disponible y a las características de la demanda en cada momento; sin embargo, opciones como ésta pertenecen al futuro, donde podrían permanecer para siempre. En el presente, el arsenal de medidas prácticas es más limitado.

\section{Bibliografia}

Acevedo, J. (1989); El servicio ejecutivo de buses de transporte público en Bogotá, trabajo presentado al IV Encuentro Técrico entre Palses Latinoamericanos sobre Transporte Urbano, La Habatra, CEPAL, mimeo.

Allpont, R. y J. Thomson (1990): Study of Mas, Rapid Transit in Developing Countries, Londres, Transport and Road Research Laboratory.

Bamford, T. y R. Allport (1990): The TRRL Metro pre-appraisal model, Urban Transport in Developing Countries, $\vee$ Conferencia sobre Transportes Urbanos en los Países en DesarroHlo, São Panlo, CODATU, septiembre.

Buleze, E. y otros (1985): Integraçăo do sistema de transporte urbano de Curitiba, trabajo presentado al Primer Eneuentro Técnico entre Países Latinoamericanos sobre Transportes Urbanos, Brasilia, Counisión Econónica para América Latina y el Caribe (CEPAL)/Empresa Brasileira dos Transportes Urbanos (EBTU), octubre, mimeo.

Comisión de Planificación de Inversiones en Infraestructura de Transporte (sin fecha): Encuesia origen destino de viajes del Gran Santiago 1991, Santiago de Chile, Secretaría Ejccutiva de la Comisión de Planificación de Inversiones en Infraestructura de Transporte (SECTRA).

El Mercurio (1996): Más estacionamientos debcrán tener las nuevas construcciones urbanas, Santiago de Chile, 27 de julio.
Empresa de Transporte de Pasajeros Metro S.A. (varios años): Memoria anual, Santiago de Chile.

Figueroa, 0. (1986): Observation des metros latino-americains, Paris, Institut National de Recherche sur les Transports et leur Securité (INRETS).

Foster, C. y M. Beesley (1963); Estimating the social benefit of constructing an underground railway in London, Journal of the Royal Statistical Society, serie A, vol. 126, parte I, Londres, Royal Statistical Society.

Knight, R. (1980): The impact of rail transit on land use: Evidence and change of perspectives, Transportation, vol, $9, \mathrm{~N}^{\circ} \mathrm{I}$, Amsterdan, Elsevier Science Publishers (B.v. North-Holland), marzo.

Jorge, M. (1994): Transponte urbano de pasajeros por trenes livíanos. Beneficios ambientales, actas del VII Congreso Latinoamericano de Transporte Publico y Urbano, Buenos Aires, Congresos Latinoamericanos sobre Transporte Páblico y Urbano, noviembre.

OCDE (Organización de Cooperación y Desarrollo Económicos) (1994): Congestion Control and Demand Management, $\mathrm{Pa}$ rís.

Pontificia Universidad Católica de Chile, Instituto de Economia (1993): Estimacióst de los betteficios sociales del Metro, Santiago de Chile, octubre. 
SOFRETU/BCEOM/CADE (Socieıé Française d'Etudes at Réalisations de Transports Urbains/Bureau Central d'Eudes pout les Equipements d'Outre-Mer/Consultora de Administración de Empresas (1968): Estudio del sistema de transporte metropolitano de Santiago de Chile, Santiago de Chile, mayo.

The Chartered Institute of Transport (1996): Better Public Transporf for Cities, Londres, junio.

Thomson, I. (1985): Los metros sudamericanos: un análisis de st evaluacion económica, Estudios urbanos y regionales, $\mathrm{N}^{\circ} 33$, Santiago de Chile, Pontificia Universidad Católica de Chile. (1996): Una evaluación crítica de algunos aspectos del de- sarrollo del sistema de transporte urbano de \$antiago, Actas del Congreso de la Sociedad Chilena de Ingeniería de Transporte, Santiago de Chile, Sociedad Chilena de Ingeniería de Transporte.

Vicente, O. y P. Brennan (1989): Los servicios diferenciales en la Región Metropolitans de Buenos Aires, trabajo presentado al IV Encuentro Técnico entre Paises Latinoamericanos sobre Transporte Urbano, La Habana, mimeo.

Younes, B. (1995): The beneftts of improving public transport: A myth or a reality?, Tronsport Reviews, vol. 15, $N^{\circ} 4$, Londres, Taylor \& Francis Ltd. 